\title{
Modeling a Rumor Propagation in Online Social Network: An Optimal Control Approach
}

\author{
Rachid Ghazzali $\mathbb{D}^{\mathrm{D}},{ }^{1}$ Amine El Bhih $\mathbb{D}^{\mathrm{D}},{ }^{1}$ Adil El Alami Laaroussi $\mathbb{D}^{1,},{ }^{1,2}$ and Mostafa Rachik ${ }^{1}$ \\ ${ }^{1}$ Laboratory of Analysis Modeling and Simulation, Department of Mathematics and Computer Science, \\ Faculty of Sciences Ben M'Sik, Hassan II University Casablanca, BP 7955, Sidi Othman, Casablanca, Morocco \\ ${ }^{2}$ Laboratory of Applied Sciences and Didactics, Higher Normal School Tetouan, Abdelmalek Essaadi University, \\ Tetouan, Morocco \\ Correspondence should be addressed to Rachid Ghazzali; ghazzalirachid@gmail.com
}

Received 29 May 2020; Accepted 14 July 2020; Published 11 August 2020

Guest Editor: Jianxin Li

Copyright ( 2020 Rachid Ghazzali et al. This is an open access article distributed under the Creative Commons Attribution License, which permits unrestricted use, distribution, and reproduction in any medium, provided the original work is properly cited.

\begin{abstract}
We propose to model the phenomenon of the spread of a rumor in social networks in this paper. From an existing SIR model, we manipulate a new one that is based on the model of cholera in order to take into account professional pages that specialize in spreading rumors. In the second part, we introduce a control strategy to fight against the diffusion of the rumor. Our main objective is to characterize the three optimal controls that minimize the number of spreader users, fake pages, and the corresponding costs. For that matter, using the maximum principle of Pontryagin, we prove the existence and we give characterization of our controls. Numerical simulations are given to concretize our approach.
\end{abstract}

\section{Introduction}

The phenomenon of rumor is a complex phenomenon that has eluded man since ancient times, where it intersects many factors and interventions, including what is natural, sociological, economic, and psychological. Communities have known over the years the emergence of many rumors that have spread widely among them; it was also the focus of interaction and analysis by the commanders of these societies throughout history [1]; human beings have fabricated rumors and disseminated them for political, economic, and social purposes [2], where they are exploited to achieve commercial profits or to achieve victories in wars by dissolving fear and surrender within the enemy or with holding confidence in their leaders. The phenomenon of rumor has known many changes in its composition, in line with the change that societies know and the development of daily life in general with the increasing use of technological instruments and modern technologies in communication within communities. This phenomenon has witnessed a dramatic rise and an increase in the speed of its spread. This increase contributes significantly to huge consequences on the other hand. The development of the phenomenon of rumors and the strength of their influence and impact within societies gave this phenomenon another dimension [3], as it became used by the media and intelligence in competition between countries and what is known as propaganda and polemic or buzz by publishing some false news in whole or in part to influence the opinions of voters by raising or decreasing the popularity of politicians [4] as happened in the elections between Trump and Hillary where Hillary was the most popular and was the favorite to win until the last weeks before the presidential election [5], where some of the specialized communication agencies published many news about Hillary contributed significantly to influence public opinion tendency to Trump, who eventually won. Jennifer et al. in their article [6] did a study in order to understand the dynamics of this exceptional campaign in which social media played a major role. The website [7] gives a variety of Trump's Tweets grouped by topic (people, places, and things Trump has insulted on Twitter). In 2018, Russian authorities have considered starting to block sites like Telegram [8] because of the danger on national security. And if some rumors arouse ridicule, such as saying that Nicolas Cage was 
Dracula, others were of great danger; to see more in this regard, we guide the reader to the beautiful book [9].

Mathematical modeling is one of the most important applications of mathematics that contribute to the representation and simulation of social, economic, biological, and ecological phenomena and convert them into mathematical equations that are formulated, studied, analyzed, and interpreted [10]. In this context, many researchers have developed different mathematical models representing the dynamics of the rumor [11] and the elements interfering with its spread [12-15], and especially [16] in the work [17], authors gave a review and a study of several mathematical models of rumor's propagation.

1.1. Related Work. In 1964, Goffman and Newill developed in their article titled "Generalization of Epidemic Theory: An Application to the Transmission of Ideas" [18] a new concept for modeling the transmission of ideas within a society based on the mathematical model SIR due to the great similarity between the two phenomena. This model was previously used to model the transmission of diseases and epidemics within communities; in the introduction of their work, the authors stated that "the process already described does not take into account the almost endless number of complexities which actually arise" [18]. Based on the previous work, Daley and Kendall in their letter titled "Epidemics and Rumors" suggested applying the previous idea to modeling the spread of rumors within communities [19]. With the development of societies and the emergence of modern technological means (transport communication), new factors have emerged that further complicate the phenomenon of rumor and contribute to the large spread of rumors; this has led many researchers to think about developing the previous model. As an example, in the work done by Luis M.A. Bettencourt et al. [20], they proposed a new model taking into account new factors by extending the SIR model to a SEIZR model with two additional compartments. In the same context and to give time factor more importance in the process of spread of the phenomenon, Laarabi et al. [21] had developed another model using a delayed rumor propagation model. This is in addition to many recent works that have recently been produced that take into account several factors involved in the development of a concept that truly simulates the dynamics of rumor propagation; to take a broader view, the reader is referred to the article [22]. With the emergence of social networks and their impacts on communication within communities where they are taking more and more space within the community, it became clear that they must be taken into account as major intervening in the spread of rumors; in this context many of the works that adopted this hypothesis have been produced. To take an idea of some of these works, see the article [23]. For example, in the work [24], authors had implemented a mathematical model in order to model the dynamics of a rumor in social network by adding three new compartments: reviewers, sharers, and collectors who are reviewing the message, collecting the message, sharing the message, or giving no response to the message, respectively, but in the work [25], the authors were limited to highlight the role of users of the network and ignore the impact of the network itself, especially the role of pages that spread the rumor within the network. The loading of false information in these pages is a source of rumor between browsers and considered as a big factor which helps in the rapid spread of rumors, such as rivers and valleys, which store bacteria and microbes and are a hotbed for the multiplication and growing bacteria that transmit diseases to humans through the use of the water of those rivers. A good example of this similarity is the cholera epidemic. In this paper and based on the previous hypothesis, we will exploit the mathematical model that has been formulated to represent the cholera epidemics [26] and combine it with the previous work model by adding a new compartment $F$ which represents page's rumor. Other models from population dynamics and optimal controls can be found in [3, 27].

Recently, a significant amount of prior works exists in the study of rumor detection in social networks. For example, in [28], the authors propose a GCN-based model for rumor detection on social media, called Bi-GCN, and they discuss several variants of $\mathrm{Bi}-\mathrm{GCN}$ to model the propagation patterns. Ma et al. in [29] discuss the same topic involving a novel approach to capture the temporal characteristics based on the time series of rumor's life cycle, for which time series modeling technique is applied to incorporate various social context information, while Han Guo et al. [30] propose a novel hierarchical neural network combined with social information (HSA-BLSTM) for rumor detection and they test their model on two real-world datasets from Weibo and Twitter demonstrating outstanding performance in both rumor detection and early detection scenarios. Li et al. [31] give another approach, the personalized influential topic search by proposing two random-walk based approaches in order to measure the influence of a topic on a query user. Moreover, Li et al. in [32] studied the problem from another side, influence maximization; the aim is to find a limited number of users which can influence the maximum number of users in social networks. Li et al. in [33] continue to improve their work by taking into account the physical locations of the users since location is an important factor in this process. The same approach was discussed by Cai et al. in [34], where they formulate a new problem of holistic influence maximization, denoted as HIM query, for targeted advertisements in a spatial social network.

1.2. Problem Definition. In [35], where the authors subdivide the population into three compartments representing the main actors in the dynamics of the propagation of a rumor, these compartments are ignorant individuals, the spreaders, and the stiflers. As we mentioned before, many agencies specialized in propaganda dissemination have become using social media to facilitate the spread of rumor and large volume of users. For this purpose, special pages are created to spread a rumor about a specific subject or target person. This page is promoted by fictitious users that are created for this purpose. They create a private network of friends; their friends are the first victims; every time they like or comment on what is posted by the page or fictitious people, this activity is displayed to all their friends or perhaps friends of 
their friends inadvertently which is promoted by this rumor passively by them, while studies indicate that the number of users of the networks is rising at a tremendous rate and it has become one of the basics in the field of communication and publicity, according to statistics [4]. It treated millions of rumors spread daily in social networks, starting from this model, and by adding an additional compartment, named $F$ as Fakes, we will build our own model which describes the propagation of a rumor through a social network. Our idea is to combine the classical model ISpSt with another mathematical model that describes the dynamics of cholera in order to highlight the importance of fake page which are specified in spreading fake news; in other words, since there is a similarity between these two phenomena, we can consider a fake page as a contaminated river which contains bacteria; these bacteria are false information in our case. We can use this model in order to describe the dynamics of the rumor between the different individuals as well as bringing out the contribution of fake pages in this process [36]. In this paper and based on the previous hypothesis, we will exploit the mathematical model that has been formulated to represent the cholera epidemics [36] and combine it with the previous work model by adding a new compartment $F$ which represents page's rumor.

In this paper, in Section 2, we propose a continuous mathematical model $I S_{p} S_{t} F$ that describes the dynamics of a population that reacts in the spread of the rumor in a social network positivity, and the boundness of the model is discussed. In 3, we present an optimal control problem for the proposed model where we give some results concerning the existence of the optimal control, and we characterize the optimal controls using the Pontryagin maximum principle in discrete time. Numerical simulations through MATLAB are given in Section 3.2. Finally, we conclude the paper in Section 4.

1.2.1. I $S_{p} S_{t} F$. In this section, we will describe our model $I S_{p} S_{t} F$ which consists of four compartments representing the subdivision of the population that reacts in the spread of the rumor in a social network. $I$, ignorant, represents users who do not know the rumor and are susceptible to be informed, $S_{p}$, spreader, represents users who spread the rumor, $S_{t}$, stifler, represents individuals who refuse to spread the rumor, and $F$, rumor's page, represents the page specialized in spreading the rumor. The compartment $I$ represents the number of users who do not know the rumor and who are susceptible to be informed; this population increases with the rate $\mu N$ which represents the new users created; an ignorant inquires about the rumor through two ways: either by consulting a specialized page in the diffusion of the rumor or directly by the contact with a spreader. Some of these users deactivate their account at a rate $\mu I$. Thus, in this compartment, we have an incoming flux equal to $\mu \mathrm{N}$ and an outgoing flux equal to $\alpha_{h} S_{p} I+\alpha_{e} I(F / \kappa+F)+\mu I$.

The compartment $S_{p}$ represents the number of people who spread the rumor either directly or by sharing one-page publications or by creating new publications. Thus, we have an incoming flux equal to $\theta\left(\alpha_{h} S_{p} I+\alpha_{e} I(F / \kappa+F)\right)$ which represents the proportion of the new users who will spread the rumor. After the contact between two spreaders, one of them decides not to diffuse the information at a rate $\gamma S_{p}^{2}$, and after the contact of a spreader and a stifler, the stifler succeeds to convince him that the information is false at a rate $\lambda S_{t} S_{p}$; after a certain period, a portion of the spreaders decide not to spread the rumor at a rate $\beta S_{p}$.

The compartment $S_{t}$ represents the number of stiflers who refuse to spread the rumor. This number increases at a rate $(1-\theta)\left(\alpha_{h} S_{p} I+\alpha_{e} I(F / \kappa+F)\right)$ which represents the portion of users who knew that the information is wrong, in addition to the flux that left the $S_{p}$ compartment $S_{p}\left(\gamma S_{p}+\right.$ $\left.\lambda S_{t}\right)+\beta S_{p}$ and decreases with the rate $\mu S_{t}$ of stiflers who have deactivated their accounts.

The compartment $F$ represents the page specialized in the diffusion of the rumor. This page contains malicious publications about the rumor; in this page, $S_{p}$ have the right to publish and share these publications at rates $\delta_{1} S_{p}$ and $\varepsilon_{2} S_{p}$, respectively, and the ignorants who consult the page also share these publications at a rate $\varepsilon_{1} I$.

The following diagram will demonstrate the flux directions of individuals among the compartments (Figure 1).

The dynamics of this model are governed by the following nonlinear system:

$$
\left\{\begin{array}{l}
\frac{\mathrm{d} I}{\mathrm{~d} t}=\mu N-\alpha_{h} S_{p} I-\alpha_{e} I \frac{F}{\kappa+F}-\mu I, \\
\frac{\mathrm{d} S_{p}}{\mathrm{~d} t}=\theta\left(\alpha_{h} S_{p} I+\alpha_{e} I \frac{F}{\kappa+F}\right)-\beta S_{p}-S_{p}\left(\gamma S_{p}+\lambda S_{t}\right), \\
\frac{\mathrm{d} S_{t}}{\mathrm{~d} t}=(1-\theta)\left(\alpha_{h} S_{p} I+\alpha_{e} I \frac{F}{\kappa+F}\right)+S_{p}\left(\gamma S_{p}+\lambda S_{t}\right)+\beta S_{p}-\mu S_{t} \\
\frac{\mathrm{d} F}{\mathrm{~d} t}=\delta_{1} S_{p}+\varepsilon_{1} I+\varepsilon_{2} S_{p} .
\end{array}\right.
$$

With initial values, $I(0), S_{p}(0), S_{t}(0)$, and $F(0)$ are nonnegatives $F / \kappa+F$ : logistic capacity (concentration of rumors).

In order to demonstrate the effectiveness of the model we have proposed, we will present a numerical simulation with the following figure so that we can see how well the model adapts to reality. Initial values are approximate data that we suggested after studying and researching some statistics about the users of social networks; the values are attached in the table.

From Figure 2, we note that there is no significant effect until the 30th day, 30 days after the launch of the rumor; the number of ignorants decreases sharply; in contrast, there is a significant rise of spreaders and the numbers of stiflers and pages is rising on average. These changes indicate that after 30 days trading rumor has become more and more due to the continuous publication of it.

\subsubsection{Model Basic Properties}

Theorem 1. If $I(0) \geq 0, S_{p}(0) \geq 0, S_{t}(0) \geq 0$, and $F(0) \geq 0$, the solutions $I(t), S_{p}(t), S_{t}(t)$, and $F(t)$ of system (1) are positive for all $t \geq 0$. 


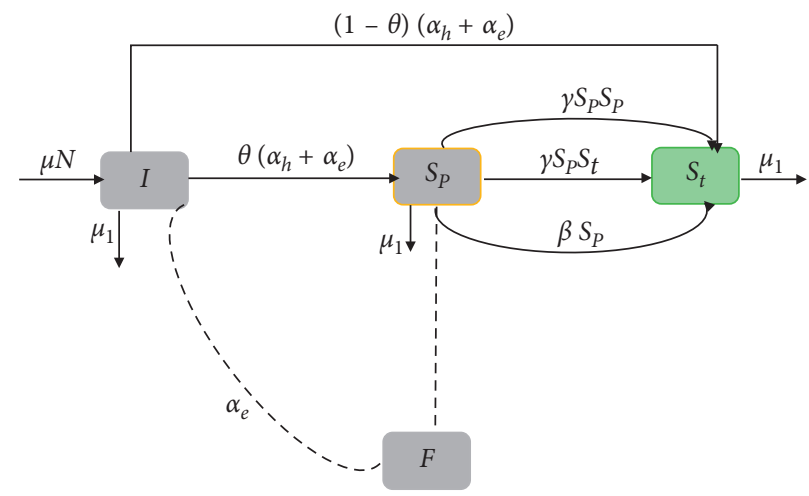

FIgURE 1: Description diagram of the rumor dynamics.

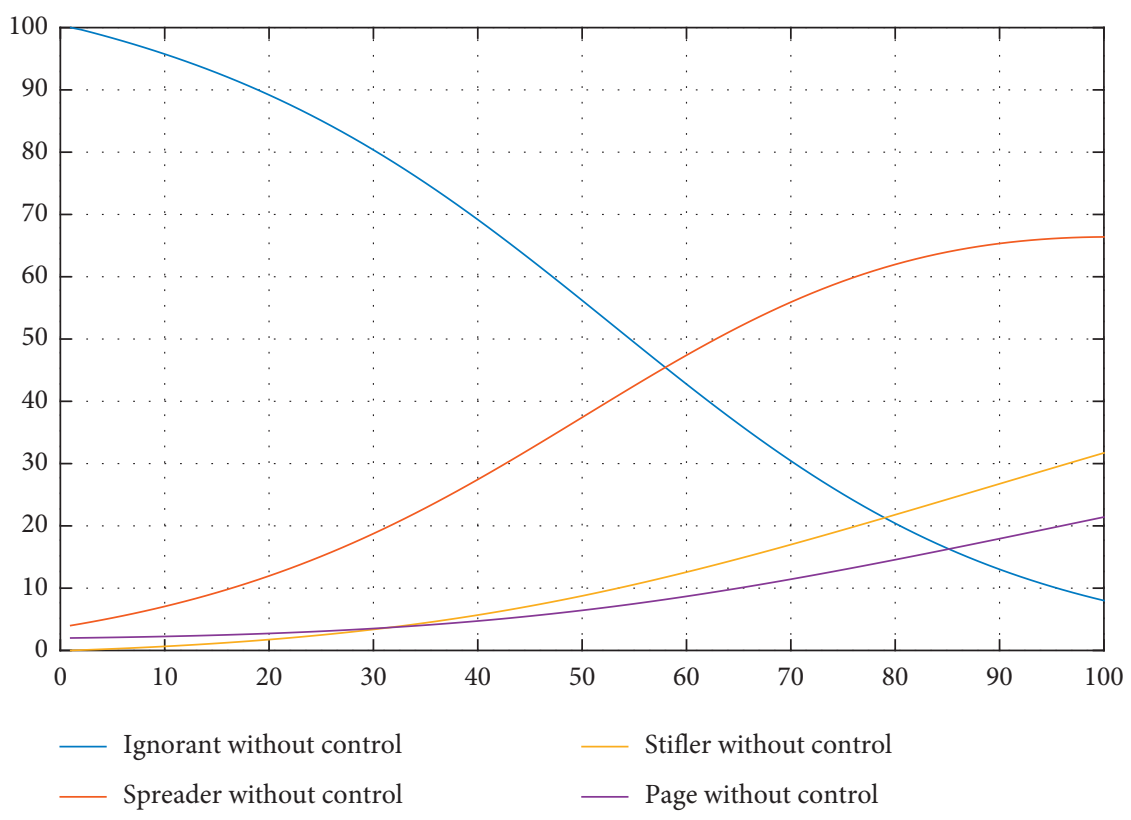

Figure 2: Dynamics without control strategy.

Proof.

$$
\begin{aligned}
\frac{\mathrm{d} I(t)}{\mathrm{d} t}= & \mu N-\alpha_{h} S_{p} I-\alpha_{e} I \frac{F}{\kappa+F}-\mu I \\
\geq & -\alpha_{h} S_{p} I-\alpha_{e} I \frac{F}{\kappa+F}-\mu I \\
& \frac{\mathrm{d} I(t)}{\mathrm{d} t}+\left(\alpha_{h} S_{p}+\alpha_{e} \frac{F}{\kappa+F}+\mu\right) I \geq 0 .
\end{aligned}
$$

where $G(t)=\alpha_{h} S_{p}+\alpha_{e}(F / \kappa+F)+\mu$. Both sides in the last inequality are multiplied by $\exp \left(\int_{0}^{t} G(s) \mathrm{d} s\right)$.

We obtain

$$
\exp \left(\int_{0}^{t} G(s) \mathrm{d} s\right) \cdot \frac{\mathrm{d} I(t)}{\mathrm{d} t}+G(t) \exp \left(\int_{0}^{t} G(s) \mathrm{d} s\right) \cdot I(t) \geq 0
$$

Then

$$
\frac{\mathrm{d}}{\mathrm{d} t}\left(I(t) \exp \left(\int_{0}^{t} G(s) \mathrm{d} s\right)\right) \geq 0
$$

Integrating this inequality from 0 to $t$ gives

$\int_{0}^{t} \frac{\mathrm{d}}{\mathrm{d} s}\left(I(s) \exp \left(\int_{0}^{t}\left(\alpha_{h} S_{p}+\alpha_{e} \frac{F}{\kappa+F}+\mu\right) \mathrm{d} s\right)\right) \mathrm{d} s \geq 0$.

Then

$$
I(t) \geq I(0) \exp \left(\int_{0}^{t}-\left(\alpha_{h} S_{p}+\alpha_{e} \frac{F}{\kappa+F}+\mu\right) \mathrm{d} s\right)
$$

$\Longrightarrow I(t) \geq 0$.

Similarly, we prove that $I(t) \geq 0, S_{p}(t) \geq 0, S_{t}(t) \geq 0$, and $F(t) \geq 0$. 


\subsubsection{Boundedness of the Solutions}

Theorem 2. The set

$$
\Omega=\left\{\frac{\left(I, S_{p}, S_{t}, F\right) \in \mathbb{R}_{+}^{4}}{0 \leq I+S_{t}+S_{p}+F \leq 1}\right\}
$$

is positively invariant under system (1) with initial conditions $I(0) \geq 0, S_{p}(0) \geq 0, S_{t}(0) \geq 0$, and $F(0) \geq 0$ being positively invariant for system (1).

\section{The Model with Controls}

Now, we introduce our controls into system (1). As control measures to fight the spread of rumor, we extend our system by including three kinds of controls $u, v$, and $w$. The first control $u$ is to tell users that the information or publication is false and contains a malicious rumor. The second control $v$ is through the admin where he deactivates an account after learning that it is fake or aimed at spreading the rumor. The last one $w$ is also applied by the admin, this time by deactivating the page intended to spread the rumor after the arrival of a certain number of complaints.

With the aim of better understanding the effects of any control measure of these strategies, we introduce three new variables: $\pi_{i}$, where $i=1,2,3, \pi_{i}=0$ in the absence of control, and $\pi_{i}=1$ in the presence of control. One has

$$
\left\{\begin{array}{l}
\frac{\mathrm{d} I}{\mathrm{~d} t}=\mu N-\alpha_{h} S_{p} I-\alpha_{e} I \frac{F}{\kappa+F}-\mu I-\pi_{1} u I \\
\frac{\mathrm{d} S_{p}}{\mathrm{~d} t}=\theta\left(\alpha_{h} S_{p} I+\alpha_{e} I \frac{F}{\kappa+F}\right)-(\beta) S_{p}-S_{p}\left(\gamma S_{p}+\lambda S_{t}\right) \\
-\pi_{2} v S_{p}, \\
\frac{\mathrm{d} S_{t}}{\mathrm{~d} t}=(1-\theta)\left(\alpha_{h} S_{p} I+\alpha_{e} I \frac{F}{\kappa+F}\right)+S_{p}\left(\gamma S_{p}+\lambda S_{t}\right) \\
+\beta S_{p}-\mu S_{t}+\pi_{1} u I, \\
\frac{\mathrm{d} F}{\mathrm{~d} t}=\delta_{1} S_{p}+\varepsilon_{1} I+\varepsilon_{2} S_{p}-\pi_{3} w F .
\end{array}\right.
$$

\section{Optimal Control Problem}

We define the objective functional as follows:

$$
J(u, v, w)=\int_{0}^{T}\left(S_{p}(t)+\frac{1}{2} A u^{2}(t)+\frac{1}{2} B v^{2}(t)+\frac{1}{2} C w^{2}(t)\right) \mathrm{d} t,
$$

where $A>0, B>0$, and $C>0$ are the cost coefficients:

$$
J\left(u^{*}, v^{*}, w^{*}\right)=\min \left\{\frac{J(u, v, w)}{(u, v, w) \in U_{\mathrm{ad}}}\right\},
$$

where $U_{\text {ad }}$ is the set of admissible controls defined by

$$
\begin{aligned}
U_{\mathrm{ad}} & =\left\{\frac{(u, v, w)}{u_{\min } \leq u(t) \leq u_{\max }}, v_{\min } \leq v(t) \leq v_{\max }, w_{\min } \leq w(t)\right. \\
& \left.\leq w_{\max } t \in[0, T]\right\},
\end{aligned}
$$

and $\left(u_{\min }, v_{\min }, w_{\min }, u_{\max }, v_{\max }, w_{\max }\right) \in(] 0,1[)^{6}$.

Theorem 3. Consider the control problem with system (8). that

There exists an optimal control $\left(u^{*}, v^{*}, w^{*}\right) \in U_{\text {ad }}^{3}$ such

$$
J\left(u^{*}, v^{*}, w^{*}\right)=\min _{(u, v, w) \in U_{\mathrm{ad}}^{3}} J\left(u^{*}, v^{*}, w^{*}\right) .
$$

If the following conditions are met:

(1) The set set of controls and the corresponding state variables is nonempty.

(2) The control set $U_{\text {ad }}$ is convex and closed.

(3) The right-hand side of the state system is bounded by a linear function in the state and control variables.

(4) The integrand $L\left(I, S_{p}, S_{t}, F, u, v, w\right)$ of the objective functional is convex on $U_{\text {ad }}$ and there exist constants $c_{1}$ and $c_{2}$ such that

$$
L\left(I, S_{-} p, S_{-} t, F, u, v, w\right) \geq c_{1}+c_{2}\left(|u|^{2}+|v|^{2}+|w|^{2}\right)^{\beta / 2} \text {. }
$$

Proof. The existence of the optimal control can be obtained using a result by Fleming and Rishel [37], checking the following step:

Condition 1: To prove that the set of controls and the corresponding state variables is nonempty, we will use a simplified version of an existence result ([38], Theorem $7)$. Let $\dot{I}=f_{I}\left(t, I, S_{p}, S_{t}, F\right), \quad \dot{S}_{p}=f_{S_{p}}\left(t, I, S_{p}, S_{t}, F\right)$, $\dot{S}_{t}=f_{S_{t}}\left(t, I, S_{p}, S_{t}, F\right)$, and $\dot{F}=f_{F}\left(t, I, S_{p}, S_{t}, F\right)$, where $f_{I}, f_{S_{p}}, f_{S_{t}}$, and $f_{F}$ form the right-hand side of the system of 8 . Let $u(t)=c_{1}, v(t)=c_{2}$, and $w(t)=c_{3}$, for some constants, and since all parameters are constants and $I, S_{p}, S_{t}$, and $F$ are continuous, then $f_{I}, f_{S_{p}}, f_{S_{t}}$, and $f_{F}$ are also continuous. Additionally, the partial derivatives $\partial f_{I} / \partial I, \partial f_{I} / \partial S_{p}, \partial f_{I} / \partial S_{t}, \partial f_{I} / \partial F, \partial f_{S_{p}} / \partial I$, $\partial f_{S_{p}} / \partial S_{p}, \quad \partial f_{S_{p}} / \partial S_{t}, \quad \partial f_{S_{p}} / \partial F, \partial f_{S_{t}} / \partial I, \quad \partial f_{S_{t}} / \partial S_{p}$, $\partial f_{S_{t}} / \partial S_{t}, \partial f_{S_{t}} / \partial F, \partial f_{F} / \partial I, \partial f_{F} / \partial S_{p}, \partial f_{F} / \partial S_{t}$, and $\partial f_{F} / \partial F$ are all continuous. Therefore, there exists a unique solution $\left(I, S_{p}, S_{t}, F\right)$ that satisfies the initial conditions. Therefore, the set of controls and the corresponding state variables is nonempty and condition 1 is satisfied.

Condition 2: By definition, $U_{\text {ad }}$ is closed. Take any control $u_{1}, u_{2} \in U_{\text {ad }}$ and $\lambda \in[0,1]$. Then $\lambda u_{1}+(1-\lambda) u_{2} \geq 0$.

Additionally, we observe that $\lambda u_{1} \leq \lambda(1-\lambda)$ $u_{2} \leq(1-\lambda)$; then $\lambda u_{1}+(1-\lambda) u_{2} \leq \lambda+(1-\lambda)=1$. 
Hence,

$0 \leq \lambda u_{1}+(1-\lambda) u_{2} \leq 1, \quad$ for all $u_{1}, u_{2} \in U_{\text {ad }}$ and $\lambda \in[0,1]$.

Therefore, $U_{\text {ad }}$ is convex and condition 2 is satisfied.

Condition 3: All the right-hand sides of equations of system are continuous, bounded above by a sum of bounded control and state, and can be written as a linear function of $u, v$, and $w$ with coefficients depending on the time and state. Therefore, condition 3 is satisfied.

Condition 4: The integrand in the objective functional (9) is convex on $U_{\mathrm{ad}}$. It rests to show that there exist constants $c_{1}, c_{2}>0$, and $\beta>1$ such that the integrand $L\left(I, S_{p}, S_{t}, F, u, v, w\right)$ of the objective functional satisfies

$$
\begin{aligned}
L\left(I, S_{p}, S_{t}, F, u, v, w\right) & =S_{p}(t)+\frac{A}{2} u^{2}(t)+\frac{B}{2} v^{2}(t)+\frac{C}{2} w^{2}(t) \\
& \geq c_{1}+c_{2}\left(|u|^{2}+|v|^{2}+|w|^{2}\right)^{\beta / 2} .
\end{aligned}
$$

The state variables are bounded; let $c_{1}=S_{p}$, $c_{2}=\inf (A / 2, B / 2, C / 2)$, and $\beta=2$; then it follows that

$$
L\left(I, S_{p}, S_{t}, F, u, v, w\right) \geq c_{1}+c_{2}\left(|u|^{2}+|v|^{2}+|w|^{2}\right)^{\beta / 2} .
$$

Then, from Fleming and Rishel [37], we conclude that there exists an optimal control.

3.1. Characterization of the Optimal Controls. In this section, we apply Pontryagin's maximum principle [26]. The key idea is introducing the adjoint function to attach the system of differential equations to the objective functional resulting in the formation of a function called the Hamiltonian. This principle converts the problem of finding the control to optimize the objective functional subject to the state of differential equations with initial condition to find the control to optimize Hamiltonian pointwise (with respect to the control).

Now, we have the Hamiltonian of the optimal problem given by

$$
\begin{aligned}
H= & S_{p}+\frac{1}{2} A u^{2}+\frac{1}{2} B v^{2}+\frac{1}{2} C w^{2}+\lambda_{1}\left(\mu N-\alpha_{h} S_{p} I-\alpha_{e} I \frac{F}{\kappa+F}-\mu I-\pi_{1} u I\right) \\
& +\lambda_{2}\left(\theta\left(\alpha_{h} S_{p} I+\alpha_{e} I \frac{F}{\kappa+F}\right)-(\beta) S_{p}-S_{p}\left(\gamma S_{p}+\lambda S_{t}\right)-\pi_{2} v S_{p}\right) \\
& +\lambda_{3}\left((1-\theta)\left(\alpha_{h} S_{p} I+\alpha_{e} I \frac{F}{\kappa+F}\right)+S_{p}\left(\gamma S_{p}+\lambda S_{t}\right)+\beta S_{p}-\mu S_{t}+\pi_{1} u I\right) \\
& +\lambda_{4}\left(\delta_{1} S_{p}+\varepsilon_{1} I+\varepsilon_{2} S_{p}-\pi_{3} w F\right) .
\end{aligned}
$$

Using Pontryagin's maximum $[26,39]$, we can say the following theorem.
Theorem 4. Let $I^{*}, S_{p}^{*}, S_{t}^{*}$, and $F^{*}$ be optimal state solutions with an associated optimal control $\left(u^{*}, v^{*}, w^{*}\right)$ for the optimal control problem. Then there exist adjoint variables $\lambda_{1}, \lambda_{2}, \lambda_{3}$, and $\lambda_{4}$ satisfying

$$
\begin{aligned}
& \lambda_{1}^{\prime}=-\left(-\lambda_{1} \alpha_{h} S_{p}-\lambda_{1} \alpha_{e} \frac{F}{\kappa+F}-\lambda_{1} \mu-\lambda_{1} \pi_{1} u+\lambda_{2} \theta\left(\alpha_{h} S_{p}+\alpha_{e} \frac{F}{\kappa+F}\right)+\lambda_{3}(1-\theta)\left(\alpha_{h} S_{p} I+\alpha_{e} I \frac{F}{\kappa+F}\right)+\lambda_{3} \pi_{1} u\right), \\
& \left.\lambda_{2}^{\prime}=-\left(1-\lambda_{1} \alpha_{h} I+\lambda_{2} \theta \alpha_{h} I-\lambda_{2}(\beta)-\lambda_{2}\left(\gamma S_{p}+\lambda S_{t}\right)-\gamma \lambda_{2} S_{p}-\pi_{2} \lambda_{2} \nu+\lambda_{3}(1-\theta) \alpha_{h} I+\lambda_{3}\left(\gamma S_{p}+\lambda S_{t}\right)\right)+\lambda_{3} \gamma S_{p}+\lambda_{4}\left(\varepsilon_{2}+\delta_{1}\right)\right), \\
& \lambda_{3}^{\prime}=-\left(-\lambda \lambda_{2} S_{p}+\lambda_{3} \lambda S_{p}-\mu \lambda_{3}\right), \\
& \lambda_{4}^{\prime}=-\left(-\lambda_{1} \alpha_{e} I \frac{\kappa}{(\kappa+F)^{2}}+\lambda_{2} \theta \alpha_{e} I \frac{\kappa}{(\kappa+F)^{2}}+\lambda_{3}(1-\theta) \alpha_{e} I \frac{\kappa}{(\kappa+F)^{2}}-\lambda_{4}-\pi_{3} \lambda_{4} w\right),
\end{aligned}
$$


with transversality conditions at time T. One has

$$
\begin{aligned}
& \lambda_{1}(T)=1, \\
& \lambda_{2}(T)=0, \\
& \lambda_{3}(T)=0, \\
& \lambda_{4}(T)=0 .
\end{aligned}
$$

Furthermore, $t \in[0, T]$ and for $\pi_{1}=\pi_{2}=\pi_{3}=1$, the optimal controls $u^{*}(t), v^{*}(t)$, and $w^{*}(t)$ are given by

$$
\begin{aligned}
& u^{*}=\min \left\{\max \left\{\frac{\pi_{1} I\left(\lambda_{1}-\lambda_{3}\right)}{A}, u_{\min }\right\}, u_{\max }\right\}, \\
& v^{*}=\min \left\{\max \left\{\frac{\lambda_{2} \pi S_{p} S_{t}}{B}, v_{\min }\right\}, v_{\max }\right\}, \\
& w^{*}=\min \left\{\max \left\{\frac{\lambda_{4} \pi_{3} F}{C}, w_{\min }\right\}, w_{\max }\right\} .
\end{aligned}
$$

Proof. For $t \in[0, T]$, the adjoint equations and transversality conditions can be obtained by using Pontryagin's maximum principle $[26,39]$ such that

$$
\begin{aligned}
\lambda_{1}^{\prime}=-\frac{\partial H}{\partial I}= & -\left(-\lambda_{1} \alpha_{h} S_{p}-\lambda_{1} \alpha_{e} \frac{F}{\kappa+F}-\lambda_{1} \mu-\lambda_{1} \pi_{1} u+\lambda_{2} \theta\left(\alpha_{h} S_{p}+\alpha_{e} \frac{F}{\kappa+F}\right)+\lambda_{3}(1-\theta)\left(\alpha_{h} S_{p} I+\alpha_{e} I \frac{F}{\kappa+F}\right)+\lambda_{3} \pi_{1} u\right) \\
\lambda_{2}^{\prime}=-\frac{\partial H}{\partial S_{p}}= & -\left(1-\lambda_{1} \alpha_{h} I+\lambda_{2} \theta \alpha_{h} I-\lambda_{2}(\beta)-\lambda_{2}\left(\gamma S_{p}+\lambda S_{t}\right)-\gamma \lambda_{2} S_{p}-\pi_{2} \lambda_{2} v+\lambda_{3}(1-\theta) \alpha_{h} I\right. \\
& \left.+\lambda_{3}\left(\gamma S_{p}+\lambda S_{t}\right)+\lambda_{3} \gamma S_{p}+\lambda_{4}\left(\varepsilon_{2}+\delta_{1}\right)\right) \\
\lambda_{3}^{\prime}=-\frac{\partial H}{\partial S_{t}}= & -\left(-\lambda \lambda_{2} S_{p}+\lambda_{3} \lambda S_{p}-\mu \lambda_{3}\right), \\
\lambda_{4}^{\prime}=-\frac{\partial H}{\partial F}= & -\left(-\lambda_{1} \alpha_{e} I \frac{\kappa}{(\kappa+F)^{2}}+\lambda_{2} \theta \alpha_{e} I \frac{\kappa}{(\kappa+F)^{2}}+\lambda_{3}(1-\theta) \alpha_{e} I \frac{\kappa}{(\kappa+F)^{2}}-\lambda_{4}-\pi_{3} \lambda_{4} w\right) .
\end{aligned}
$$

For $t \in[0, T]$, the optimal controls $u^{*}, v^{*}$, and $w^{*}$ can be solved from the optimality condition:

$$
\begin{aligned}
& \frac{\partial H}{\partial u}=A u-\pi_{1} \lambda_{1} I+\lambda_{3} \pi_{1} I=0 \Longleftrightarrow u=\frac{\pi_{1} I\left(\lambda_{1}-\lambda_{3}\right)}{A}, \\
& \frac{\partial H}{\partial v}=B v-\lambda_{2} \pi v S_{p} S_{t}=0 \Longleftrightarrow v=\frac{\lambda_{2} \pi S_{p} S_{t}}{B}, \\
& \frac{\partial H}{\partial w}=C w-\lambda_{4} \pi_{3} F=0 \Longleftrightarrow w=\frac{\lambda_{4} \pi_{3} F}{C} .
\end{aligned}
$$

For the bounds in $U_{\text {ad }}$ of the controls, it is easy to obtain $u^{*}, v^{*}$, and $w^{*}$ given by

$$
\frac{\pi_{3} F}{C))}
$$

$$
\begin{aligned}
& u^{*}=\min \left(1, \max \left(0, \frac{\pi_{1} I\left(\lambda_{1}-\lambda_{3}\right)}{A}\right)\right), \\
& v^{*}=\min \left(1, \max \left(0, \frac{\lambda_{2} \pi S_{p} S_{t}}{B}\right)\right), \\
& w^{*}=\min \left(1, \max \left(0, \lambda_{4}\right.\right.
\end{aligned}
$$

However, if $\pi_{i}=0$ where $i=1,2,3$, the controls attached to this case will be eliminated and removed. 


\subsection{Numerical Simulation}

3.2.1. Algorithm. In this section, we present the results obtained by solving numerically the optimality system. This system consists of the state system, adjoint system, initial and final time conditions, and the control characterization. So, the optimality system is given by the following: step 1. $I_{0}=i_{0}, \quad S_{p 0}=s_{p 0}, \quad S_{t 0}=s_{t 0}, \quad F_{0}=f_{0}$, $\lambda_{2, N}=\lambda_{3, N}=\lambda_{4, N}=0, \lambda_{1, N}=A_{N}$, and given $u_{k ; 0}^{*}, v_{k ; 0}^{*}$, $w_{k ; 0}^{*}$

step 2. for $k=0 ; 1 ; \ldots ; N-1$ do:

$$
\begin{aligned}
& I_{k+1}=\mu N-\alpha_{h} S_{p, k} I_{k}-\alpha_{e} I_{k} \frac{F_{k}}{\kappa+F_{k}}-\mu_{1} I_{k} \\
& S_{p(k+1)}=\theta\left(\alpha_{h} S_{p, k} I_{k}+\alpha_{e} I_{k} \frac{F_{k}}{\kappa+F_{k}}\right)-(\mu+\beta) S_{p, k}-\gamma S_{p, k}\left(S_{p, k}+S_{t, k}\right) \\
& S_{p(k+1)}=(1-\theta)\left(\alpha_{h} S_{p, k} I_{k}+\alpha_{e} I_{k} \frac{F_{k}}{\kappa+F_{k}}\right)+\gamma S_{t, k}\left(S_{p, k}+S_{t, k}\right)+\beta S_{p, k}-\mu S_{t, k} \text {, } \\
& F_{k+1}=\varepsilon_{1} N+\varepsilon_{2} S_{p, k}-\mu_{2} F_{k} \\
& \vdots \quad \vdots \\
& \vdots \quad \vdots \\
& \lambda_{1, T-k}=\lambda_{1, T-k+1}\left(1-\alpha_{h} S_{p, k}-\alpha_{e} \frac{F_{k}}{\kappa+F_{k}}-\mu_{1}\right)+\lambda_{2, T-k+1} \theta\left(\alpha_{h} S_{p, k}+\alpha_{e} \frac{F_{k}}{\kappa+F_{k}}\right)+\lambda_{3, T-k+1}(1-\theta)\left(\alpha_{h} S_{p, k}+\alpha_{e} \frac{F_{k}}{\kappa+F_{k}}\right), \\
& \lambda_{2, T-k}=1+\lambda_{1, T-k+1}\left(-\alpha_{h} I_{k}\right)+\lambda_{2, T-k+1}\left(1+\theta \alpha_{h} I_{k}-(\mu+\beta)-2 \gamma\right)+\lambda_{3, T-k+1}\left[(1-\theta)\left(\alpha_{h} S_{p, k}\right)+2 \gamma+\beta\right]+\lambda_{4, T-k+1} \varepsilon_{2} \text {, } \\
& \lambda_{3, T-k}=\lambda_{2, T-k+1}\left[-\gamma S_{p, k} S_{p, k}\right]+\lambda_{3, T-k+1}\left[1+\gamma S_{p, k}-\mu\right], \\
& \lambda_{4, T-k}=\lambda_{1, T-k+1}\left[-\alpha_{e} I_{k} \frac{\kappa}{\left(\kappa+F_{k}\right)^{2}}\right]+\lambda_{2, T-k+1} \theta\left[\alpha_{e} I_{k} \frac{\kappa}{\left(\kappa+F_{k}\right)^{2}}\right]+\lambda_{3, T-k+1}(1-\theta)\left[\alpha_{e} I_{k} \frac{\kappa}{\left(\kappa+F_{k}\right)^{2}}\right]+\lambda_{4, T-k+1}\left(1-\mu_{2}\right) \text {, } \\
& u_{k+1}=\min \left[b, \max \left(a, \frac{1}{A_{k}}\left(\lambda_{1, T-k+1} I_{k}-\lambda_{3, T-k+1} \gamma S_{p, k} I_{k}\right)\right)\right] \text {, } \\
& v_{k+1}=\min \left[d, \max \left(c, \frac{1}{B_{k}}\left(-\lambda_{2, T-k+1} \gamma\left(S_{p, k}\right)^{2} S_{t, k}\right)\right)\right] \text {, } \\
& w_{k+1}=\min \left[f, \max \left(e, \frac{1}{C_{k}}\left(-\lambda_{4, T-k+1} F_{k}\right)\right)\right] \text {, }
\end{aligned}
$$

end for

step 3. for $k=0 ; 1 ; \ldots ; N$; write:

$$
\begin{aligned}
I_{k}^{*} & =I_{k}, \\
S_{p, k}^{*} & =S_{p, k}, \\
S_{t, k}^{*} & =S_{t, k}, \\
F_{k}^{*} & =F_{k}, \\
u_{k}^{*} & =u_{k}, \\
v_{k}^{*} & =v_{k}, \\
w_{k}^{*} & =w_{k},
\end{aligned}
$$

end for.
In this formulation, there were initial conditions for the state variables and terminal conditions for the adjoints. That is, the optimality system is a two-point boundary value problem with separated boundary conditions at time steps $k=0$ and $k=N$. We solve the optimality system by an iterative method with forward solving of the state system followed by backward solving of the adjoint system. We start with an initial guess for the controls at the first iteration, and then before the next iteration, we update the controls by using the characterization. We continue until convergence of successive iterates is achieved.

In this paragraph, we give numerical simulation to highlight the effectiveness of the strategy that we have developed in the framework of eliminating the rumor and limit its spread; the initial values are the same as in 
TABLE 1: Rumor model parameters and values.

\begin{tabular}{lcc}
\hline Parameter & Description & Value \\
\hline$\alpha_{h}$ & The proportion of ignorants who become spreaders after discussing with a spreader & $(0.05 /$ day) \\
$\alpha_{e}$ & The proportion of ignorants who become spreaders after consulting a page of rumor & $(0.07 /$ day) \\
$\theta$ & The proportion of ignorants who become spreaders & $(0.85 /$ day) \\
$(1-\theta)$ & The proportion of ignorants who become stiflers & $(0.15 /$ day) \\
$\mu$ & New users and deactivated users & 0.08 \\
$\beta$ & The proportion of spreaders who become stiflers & $(0.2 /$ day) \\
$\gamma$ & The proportion of spreaders who become stiflers after contacting another spreader & $(0.005 /$ day) \\
$\varepsilon_{1}$ & The rate of shared publications of rumor page by ignorants & $(0.05 /$ day) \\
$\varepsilon_{2}$ & The rate of shared publications of rumor page by spreaders & $0.1 /$ day \\
$\delta_{1}$ & New rumor pages created by spreaders & $0.4 /$ day \\
\hline
\end{tabular}

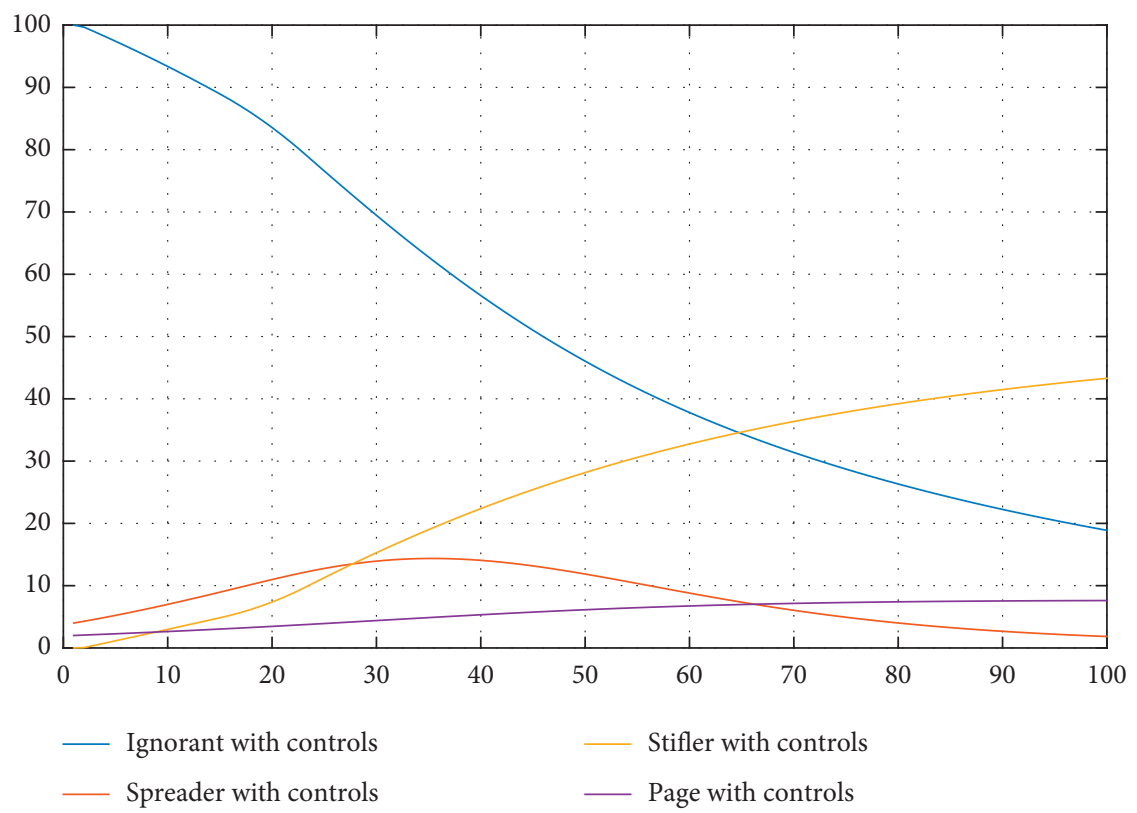

FIgURE 3: Dynamics with controls $u$, $v$, and $w$.

Table 1; with regard to other initial values, they proposed values after a statistical study.

From Figure 3, we note that, after 30 days of the implementation of the preventive strategy, effect begins to appear where we can see again that the sharp decline in the number can be observed, but this time accompanied by a high number and a gradual decline for both $S_{p}$ and $F$. This confirms that the proposed strategy in theory is paying off.

In this paragraph and with the aim of obtaining more accurate information about the impact of each control separately, we will develop a preventive strategy by applying each of them individually; numerical analysis will show us the effectiveness of each prevention strategy.

3.2.2. Case 1: Applying Only Control $u$. Since it will be applied to ignorant individuals, we will be limited to displaying and comparing the curves of $S_{p}$ and $S_{t}$ in both cases with and without control strategy. We observe from Figure 4 that, 30 days after the implementation of the strategy, the impact will start to appear as we note that the number will gradually decrease until it stabilizes at 45 . On the other hand, the number of $S_{t}$ will suddenly start to rise from about the first day. This change is probably due to the fact that the control is aimed at telling the ignorant people to turn to stifler ones. In this way, we win a number of people in the fight against the spread of false news.

3.2.3. Case 2: Applying Only Control v. Here, we will implement only control $v$, noting through Figure 5 that the effect of the strategy will appear after 20 days on the number of $S_{p}$ as the number will gradually decrease. This rapid change is attributed to the fact that control directly targets this group. The number of $I$ and $S_{t}$ change begins to appear after 40 to 50 days as the number of ignorant individuals is relatively high after the implementation of the strategy in parallel with the fact that there is a relatively high number of $S_{t}$ people which is considered logical and simulates reality since the high number of $S_{t}$ is the one that caused the number of $I$ to be raised due to their transfer of the correct information to them. 


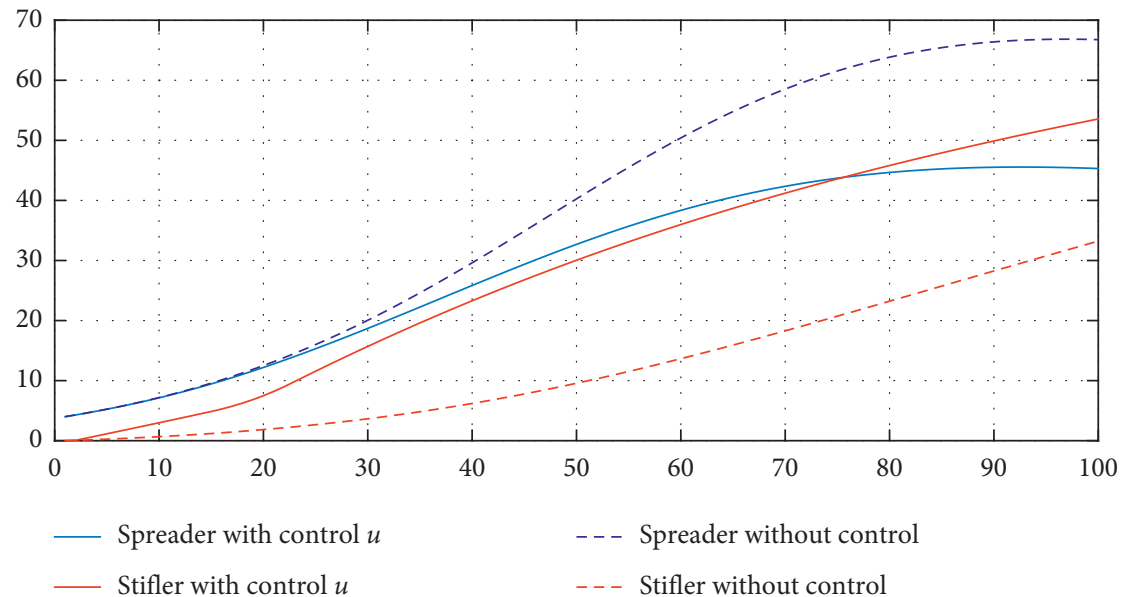

Figure 4: Dynamics with controls $u$.

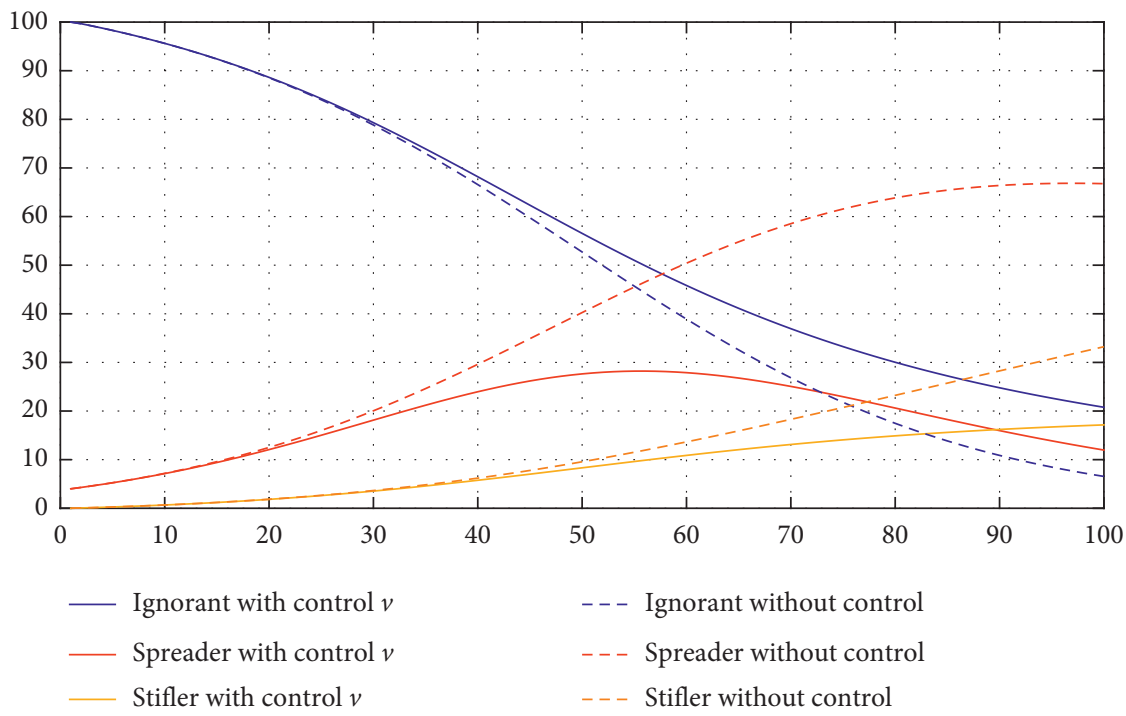

FIgURe 5: Dynamics with controls $v$.

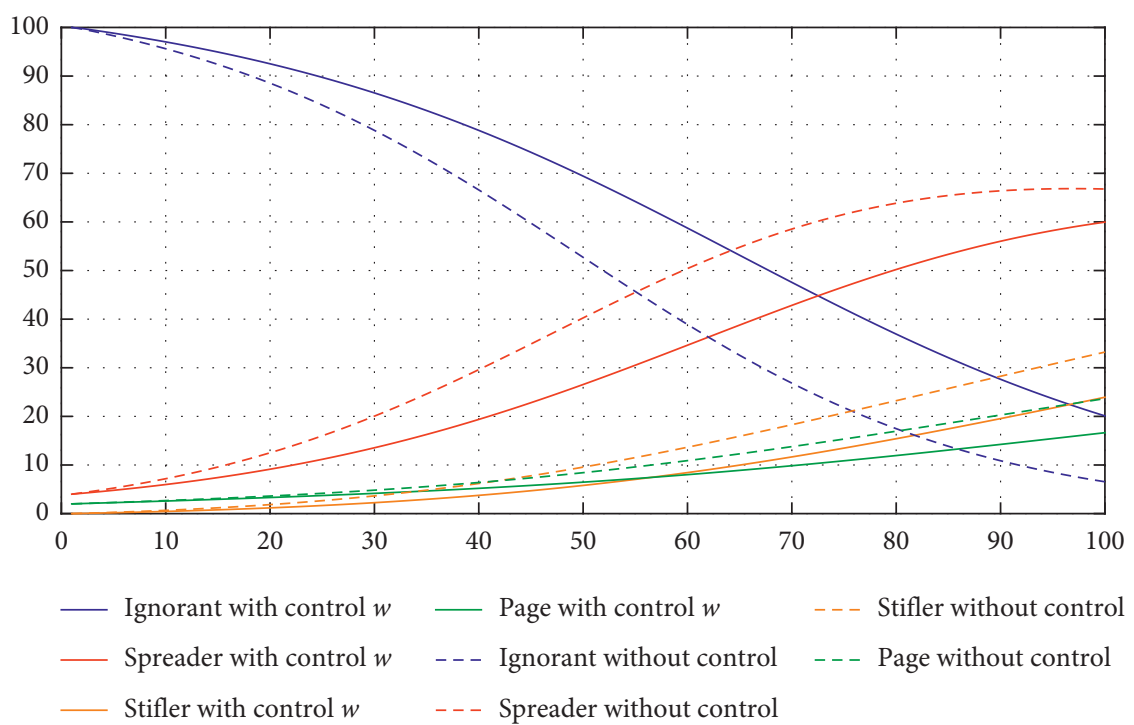

Figure 6: Dynamics with controls $w$. 
3.2.4. Case 3: Applying Only Control $w$. In the last case, we apply only control $w$. Note through Figure 6 that the effect begins immediately (after about 5 days); for the number of ignorants, we note that there is a gradual decline which is less than the number of ignorant in the absence of control; this is mainly due to low sources of rumors; the same observation is for spreader's number; it rises relatively weaker and is stabilizing at 60 thousand ones; the same thing gets with the number of stiflers where we observe that it rises up to the limits of 25 thousands; the number of pages further rises until the value reaches 15 . These observations clearly illustrate the importance of this strategy in the fight against the spread of rumors, where we see the speed of its impact and also to reach the stage of stability after its application since it targets the sources of rumors directly.

\section{Conclusion}

In this paper, we give a new simple mathematical model which describes the dynamics of rumor propagation through social network. The model is based on two compartmental models by combining them in order to take into account more factors that are involved in the dynamic. Three control strategies were introduced, and referring to the introduction of three new variables $\pi_{i}, i=1,2,3$, we could study and combine several scenarios in order to see the impact and the effect of each one of these controls on the reduction of the rumor spread. The goal is achieved and the numerical resolution of the system with difference equations as well as the numerical simulations enabled us to compare and see the difference between each scenario in a concrete way. The purpose of the work is achieved and we have proved the effectiveness of our strategy and its importance in fighting the spread of any rumor throughout any social network.

\section{Data Availability}

The disciplinary data used to support the findings of this study have been deposited in the Network Repository (http://www.networkrepository.com).

\section{Conflicts of Interest}

The authors declare that they have no conflicts of interest.

\section{Acknowledgments}

The research reported in this paper was supported by the Moroccan Systems Theory Network.

\section{References}

[1] G. W. Allport and L. Postman, The Psychology of Rumor, Holt Rinehart \& Winston, New York, NY, USA, 1947.

[2] P. Bordia and N. Difonzo, "Psychological motivations in rumor spread," Analysis of Commercial Rumors from the Perspective of Marketing Managers: Rumor Prevalence, Effects, and Control Tactics, pp. 87-101, 2005.

[3] R. Ghazzali, A. E. A. Laaroussi, A. EL Bhih, and M. Rachik, "On the control of a reaction-diffusion system: a class of SIR distributed parameter systems," International Journal of Dynamics and Control, vol. 7, no. 3, pp. 1021-1034, 2019.

[4] https://zephoria.com/top-15-valuable-facebook-statistics/.

[5] L. J. Sabato, K. Kondik, and G. Skelley, Republicans 2016: What to Do with the Donald?, 2015, http://centerforpolitics.org/crystalball/ articles/republicans-2016-what-to-do-withthe-donald/.

[6] J. Fromm, S. Melzer, B. Ross, and S. Stieglitz, Trump versus Clinton-Twitter Communication during the US Primaries, Palgrave Macmillan, London, UK, 2016.

[7] C. Lee and K. Quealy, The 337 People, Places and Things Donald Trump Has Insulted on Twitter: A Complete List, 2016, https:// www.nytimes.com/interactive/2016/01/28/upshot/donald-trumptwitter-insults.html.

[8] https://www.reuters.com/article/us-russia-telegramblocking/russia-starts-blocking-telegram-messengeridUSKBN1HN13J.

[9] R. E. Bartholomew and P. Hassall, A Colorful History of Popular Delusions, Prometheus Books, Buffalo, NY, USA, 2015.

[10] D. P. Maki and M. Thompson, Mathematical Models and Applications, Prentice-Hall, Englewood cliffs, NJ, USA, 1974.

[11] A. Rapoport and L. I. Rebhun, "On the mathematical theory of rumor spread," The Bulletin of Mathematical Biophysics, vol. 14, no. 4, pp. 375-383, 1952.

[12] M. H. Zheng, L. Y. Lv, and M. Zhao, "Spreading in online social networks: the role of social reinforcement," Physical Review E, vol. 88, no. 1, Article ID 012818, 2013.

[13] L. Fan, W. Wu, X. Zhai, K. Xing, W. Lee, and D.-Z. Du, "Maximizing rumor containment in social networks with constrained time," Social Network Analysis and Mining, vol. 4, no. 1, 2014.

[14] A. Jain, V. Borkar, and D. Garg, "Fast rumor source identification via random walks," Social Network Analysis and Mining, vol. 6, no. 1, p. 62, 2016.

[15] S. Santhoshkumar and L. D. Dhinesh Babu, "Earlier detection of rumors in online social networks using certainty-factorbased convolutional neural networks," Social Network Analysis and Mining, vol. 10, no. 1, p. 20, 2020.

[16] D. J. Daley and D. G. Kendall, "Epidemics and Rumours," Nature, vol. 204, p. 1118, 1964.

[17] M. Z. Ndii, E. Carnia, and A. K. Supriatna, "Mathematical models for the spread of rumors: a review," in Proceedings of the 6th International Congress on Interdisciplinary Behavior and Social Sciences (ICIBSoS 2017), Bali, Indonesia, July 2018.

[18] W. O. Kermack and A. G. McKendrick, "A contribution to the mathematical theory of epidemics," Proceedings of the Royal Society of London. Series A, vol. 115, pp. 700-721, 1927.

[19] W. Goffman and V. A. Newill, "Generalization of epidemic theory: an application to the transmission of ideas," Nature, vol. 204, no. 4955, pp. 225-228, 1964.

[20] D. J. Daley and D. G. Kendall, "Statistics epidemics and rumours," Nature, vol. 204, p. 1964.

[21] H. Laarabi, A. Abta, M. Rachik, and J. Bouyaghroumni, "Stability analysis of a delayed rumor propagation model," Differential Equations and Dynamical Systems, vol. 24, no. 4, pp. $407-415,2016$.

[22] R. Zhang and D. Li, "Rumor propagation on networks with community structure," Physica A: Statistical Mechanics and its Applications, vol. 483, pp. 375-385, 2017.

[23] J. Jia and W. Wu, "A rumor transmission model with incubation in social networks," Physica A: Statistical Mechanics and its Applications, vol. 491, pp. 453-462, 2018.

[24] Y. Hu, Q. Pan, W. Hou, and M. He, "Rumor spreading model with the different attitudes towards rumors," Physica A: 
Statistical Mechanics and its Applications, vol. 502, pp. 331344, 2018.

[25] A. G. Hakim, H. C. Favre1, and D. A. Zighed, Information Diffusion in Online Social Networks: A Survey SIGMOD Record, vol. 42, no. 2, 2013.

[26] L. S. Pontryagin, V. G. Boltyanskii, R. V. Gamkrelidze, and E. Mishchenko, The Mathematical Theory of Optimal Processes (International Series of Monographs in Pure and Applied Mathematics), Interscience, New York, NY, USA, 1962.

[27] A. El Bhih, Y. Benfatah, S. Ben Rhila, M. Rachik, and A. El Alami Laaroussi, "A spatiotemporal prey-predator discrete model and optimal controls for environmental sustainability in the multifishing areas of Morocco," Discrete Dynamics in Nature and Society, vol. 2020, pp. 1-18, 2020.

[28] B. Tian, X. Xiao, T. Xu et al., Rumor Detection on Social Media with Bi-directional Graph Convolutional Networks, http:// arxiv.org/abs/2001.06362, 2020.

[29] J. Ma, W. Gao, Z. Wei, Y. Lu, and K.-F. Wong, "Detect rumors using time series of social context information on microblogging websites," in Proceedings of the 24th ACM International on Conference on Information and Knowledge Management-CIKM'15, Melbourne, Australia, 2015.

[30] G. Birkhoand and G. C. Rota, Ordinary Dierential Equations, John Wiley \& Sons, New York, NY, USA, 4th edition, 1989.

[31] H. Guo, J. Cao, Y. Zhang, J. Guo, and J. Li, "Rumor detection with hierarchical social attention network," in Proceedings of the 27th ACM International Conference on Information and Knowledge Management-CIKM'18, Torino, Italy, October 2018.

[32] J. Li, T. Cai, K. Deng, X. Wang, T. Sellis, and F. Xia, "Community-diversified influence maximization in social networks," Information Systems, vol. 92, p. 101522, 2020.

[33] J. Li, T. Sellis, J. S. Culpepper, Z. He, C. Liu, and J. Wang, "Geo-social influence spanning maximization," IEEE Transactions on Knowledge and Data Engineering, vol. 29, no. 8, pp. 1653-1666, 2017.

[34] T. Cai, J. Li, A. S. Mian, R. Li, T. Sellis, and J. X. Yu, "Targetaware holistic influence maximization in spatial social networks," IEEE Transactions on Knowledge and Data Engineering, p. 1, 2020.

[35] L. Zhao, H. Cui, X. Qiu, X. Wang, and J. Wang, "SIR rumor spreading model in the new media age," Physica A: Statistical Mechanics and its Applications, vol. 392, no. 4, pp. 995-1003, 2013.

[36] J. Wang and C. Modnak, "Modeling cholera dynamics with controls canadian applied mathematics quarterly," , vol. 19, no. 3, 2011.

[37] W. H. Fleming and R. W. Rishel, Deterministic and Stochastic Optimal Control, Springer, New York, NY, USA, 1975.

[38] W. E. Boyce and R. C. DiPrima, Elementary Differential Equations and Boundary Value Problems, John Wiley \& Sons, New York, NY, USA, 2009.

[39] V. Guibout and A. M. Bloch, "A discrete maximum principle for solving optimal control problems," in Proceedings of the 2004 43rd IEEE Conference on Decision and Control (CDC), vol. 2, pp. 1806-1811, Atlantis, Bahamas, December 2004. 